\title{
Generation Of 3D City Model From Multi-Sensor Geo-Spatial Image Data And It's Visualization
}

\author{
V. NaveenChandra ${ }^{1}$ and Dr. N. V. Rao ${ }^{2}$ \\ ${ }^{1}$ CVR College of Engineering, Department of CSE, Ibrahimpatan, R.R.District, A.P., India \\ Email: vnc526@gmail.com \\ ${ }^{2}$ CVR College of Engineering, Department of CSEIT, Ibrahimpatan, R.R.District, A.P., India \\ Email: nvr@ieee.org
}

\begin{abstract}
Generation of 3D city models has been evolving to be one of the most relevant and active research topics as many application areas require them. Planning of Telecommunications, Urban development, Managing disasters, Prevention of Hazards, City design, developing TV games, etc., are some of the application areas where 3D city models are precisely and accurately needed. Several concepts from Geo-Information System (GIS) were taken for transmitting traditional 2D models to 3D City Models.

The paper describes the Generation of 3D city model in a Semi-Automated Approach. Different multi-sensor data/Images were studied and analyzed, and the images were pre-processed using image processing techniques. The 3D city model generation in semi-Automated approach involves development of prototype software which eventually can be utilized in the Automatic generation of 3D city model. The entire prototype software is designed and developed using MATLAB platform. The generated 3D city model is represented or visualized using suitable visualization techniques.
\end{abstract}

Index Terms - GIS, Photogrammetry, LIDAR, GPU, DEM, DSM, DTM, Aerial photography.

\section{INTRODUCTION}

3D city model graphically simplifies complicated concepts which are difficult to visualize. The technical standards and commercial possibilities are high in 3D representation, as in today's world people are smart enough to use 3D city model in tremendous technical, environmental and commercial aspects such as marketing, tourism, city planning, traffic management, navigation, noise propagation and much more.

3D city model consists of buildings, streets, terrain, vegetations and other man-made objects. Modeling of buildings is the main workload and is clear that photogrammetry is able to provide a means to collect the required 3D information. Generation of 3D city model is broadly classified into two large classes, namely Automatic systems [1] and Semi-Automatic systems [2]. In Automatic systems, 3D city models are generated without any human interaction. The system itself detects all the objects such as roads, trees, grass, rivers, hills, etc. whereas in Semi-Automatic approach user manually selects the buildings, towers, trees, etc and based on the selection 3D city model is generated.

In this paper we have presented 3D city model developed in semi-automated approach. The paper focus on research work for generation of 3D city model from geo-spatial data collected from multiple sources. Initially, different multi-sensor data and images were analyzed and pre-processed. Then, 3D city model was constructed and is represented using suitable visualization techniques. The prototype is designed and developed using MATLAB platform and is optimized using GPU (Graphical Processing Units) based computing techniques. The prototype software developed here will be eventually utilized to generate automatic systems.

Visualization through visual imagery has been an effective way to communicate both abstract and concrete ideas since the dawn of man. The tool used here for visualization of 3D city model is ParaView, an opensource, multi-platform data analysis and visualization application. 3D city buildings generated in this approach is stored as a grid file in 'VTK' (Visualization Tool Kit) format. VTK is an open-source, freely available software for 3D computer graphics, image processing and visualization.

Geo-Spatial Image data refers to different kinds of data such as Aerial Photography, Topographic Images, Digital Surface Model (DSM) files, Digital Terrain Model (DTM) files, Digital Elevation Model (DEM) files, LIDAR (Light Detection and Ranging) data, Satellite Imagery and TLS (Three Line Scanner) Image information.

\section{A. Abbreviations and Acronyms}

GIS (Geographical Information System):GIS is a system of systems collecting, processing, and analyzing Spatio-Temporal information regarding earth features [6]. It involves people preparing data, the system (I/O devices, computing platforms and networks) and Users using the system.

DEM (Digital Elevation Model):DEM is a digital model for 3D representation of terrain's surface.

DSM (Digital Surface Model):DSM represents the earth's surface and includes all objects in it.

DTM (Digital Terrain Model):DTM represents the bare ground surface without any objects like plants and buildings.

LIDAR (Light Detection and Ranging):It is an optical remote sensing technology that can measure the distance to, or other properties of a target by illuminating the target with light [13], often using pulses from a laser.

TLS (Three Line Scanner):Three Line Scanner (TLS) is an optical sensor for aerial survey. TLS is composed of three linear CCD arranged in parallel, and it can acquire three images of each direction (Forward, Nadir and Backward) at the same time. Orienting them on an aircraft perpendicularly to flight direction, and scanning a 
ground plane, a treble stereo image of a ground object can be acquired [3].

GPU (Graphics Processing Unit):A graphics processing unit or GPU (also occasionally called visual processing unit (VPU)) is a specialized electronic circuit designed to rapidly manipulate and alter memory in such a way so as to accelerate the building of images in a frame buffer intended for output to a display. GPUs are used in embedded systems, mobile phones, personal computers, workstations, and game consoles.

CSG (Constructive Solid Geometry):Geometric methods to build roofs of buildings such as Flat, Desk, Gable and Hip roofs.

Apart from the above terms and definitions, some of the keywords include Aerial photography, Satellite Imagery, Topographic Images, Terrestrial images, Stereo Image Data, etc., comes under Geo-Spatial data.

\section{CONSTRUCTING 3D CITY MODEL}

3D city models are digital representations of the Earth's surface and related objects belonging to urban areas (like cities, factories, buildings, etc). MATLAB supports various forms of Image formats. The Input Image can be a TIF, BMP, JPEG, PNG, GIF, etc,. MATLAB processes it and can produce preferable output. A GUI is designed for processing of images and to generate the expected model.

\section{A. Selection of Buildings}

Consider any aerial image and select the corner points of buildings. Based on the markings, draw the outlines of buildings to verify whether the lines are interacting with the geometrical shapes of buildings. If not then erase the old markings and new ones are replaced.

\section{B. Mapping the boundaries with building edges}

As we get the exact outlines of buildings, we will extract the boundary lines of buildings and are mapped with the buildings edges that we get by implementing edge detection techniques such as sobel's method, canny's method and robert's method. These algorithms give us fine line extraction [2] of buildings which is the input for generating 3D city model.

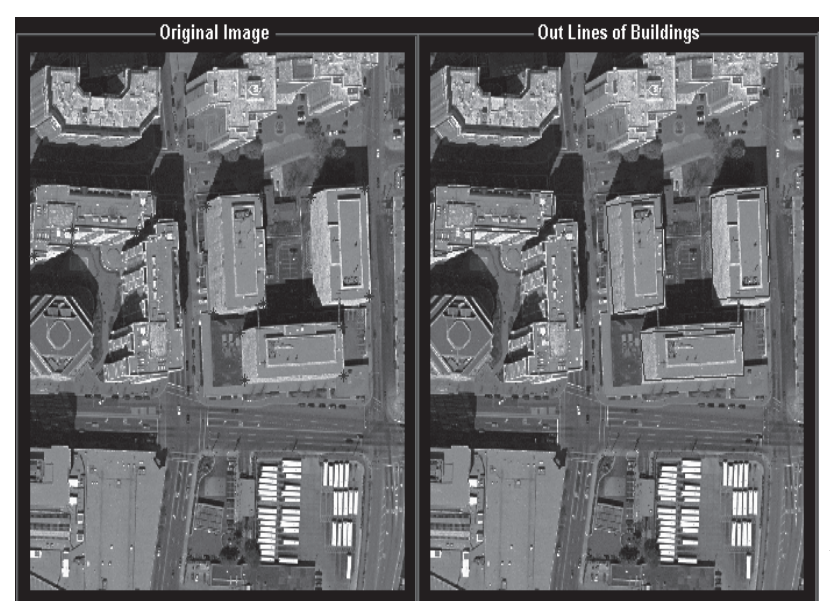

Figure 1. Markings of buildings and their outlines

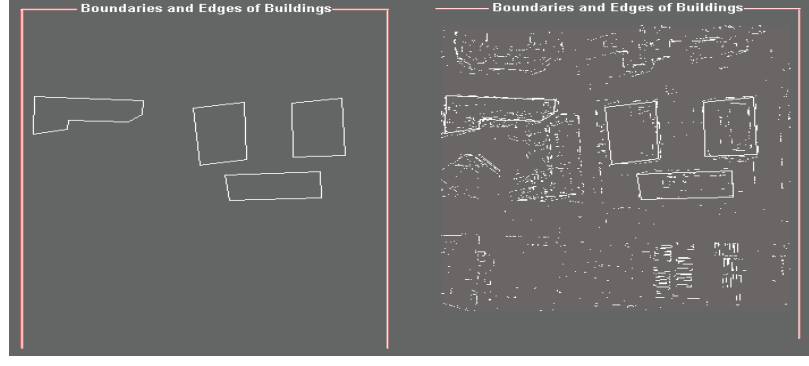

Figure 2. Mapping boundaries and edges (Robert's edge detection)

\section{Generating $3 D$ city model}

Digital Elevation Model provides the height data for a particular area on which we are generating 3D model. For the time being assuming a particular height for all buildings, 3D city model is generated using fine line boundaries which are the efficient input. 2D image is kept as a surface and on top of that 3D projections were drawn according to the boundaries obtained. 3D model from a traditional 2D image is successfully completed.

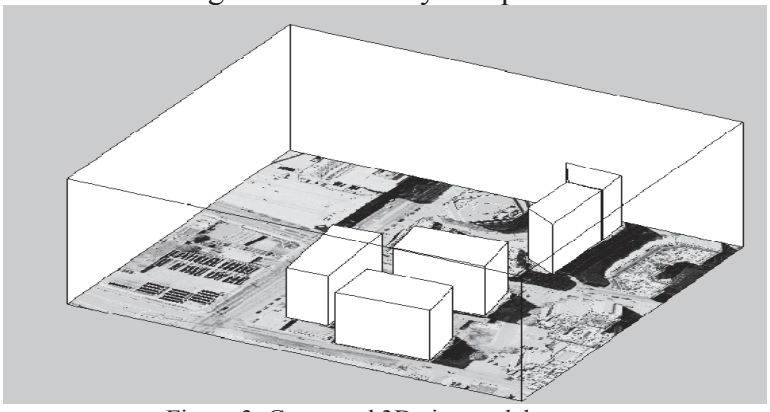

Figure 3. Generated 3D city model

\section{GENERATING A GRID FILE (VTK) AND VisuALIZATION OF 3D CiTY MODEL}

\section{A. Grid file generation}

Assume a 3D grid with our 2D image as its base with some respective value of height. As we have developed $3 \mathrm{D}$ city model by developing the building blocks on the top of $2 \mathrm{D}$ image, so the points at which the buildings are touching the $3 \mathrm{D}$ grid were considered and stored in an external file ('.vtk' file). VTK has an extensive information visualization framework, having a suite of 3D interaction widgets, It supports parallel processing, and integrates with various databases on GUI toolkits such as Qt and Tk. VTK is a cross-platform and runs on Linux, Windows, Mac and Unix platforms.

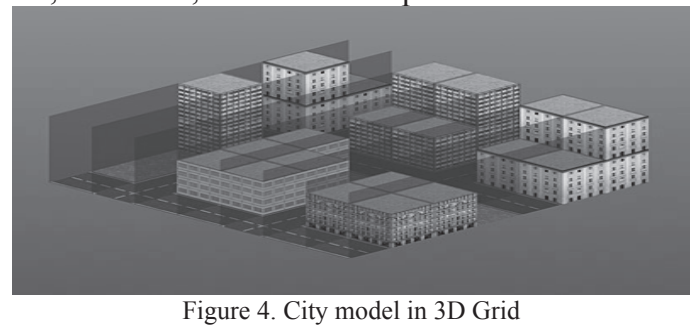

\section{B. Visualization using ParaView}

The generated 'vtk' file can be visualized using visualization tool ParaView. ParaView is an open-source, 
multi-platform data analysis and visualization application. ParaView users can quickly build visualizations to analyze their data using qualitative and quantitative techniques. The data exploration can be done interactively in 3D or programmatically using ParaView's batch processing capabilities. When the 'vtk' file is opened in ParaView the buildings of City model are visualized. This 'vtk' file is subsequently used for various applications

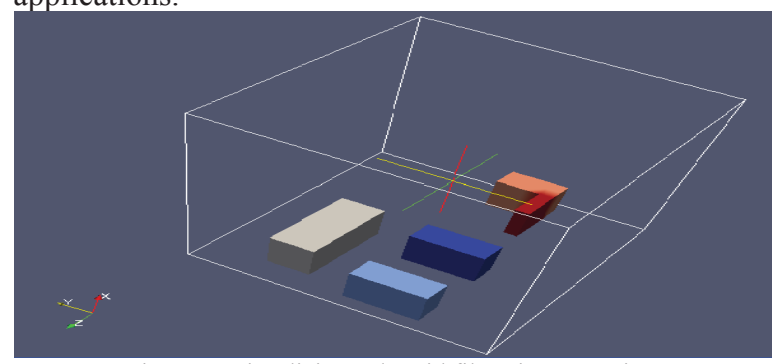

Figure 5. Visualizing 'vtk' grid file using ParaView

\section{CONCLUSIONS}

Generation of 3D city model from Aerial images in Semi-Automated approach is successfully done. The prototype software which is built here will be utilized for Automatic Generation of 3D city Model. The 'vtk' file generated is the efficient input for effective visualization of 3D city Model. The external file (vtk) is portable and hence 'vtk' file generated for any area can be easily carried and it can also be used in many applications such as traffic management, tourism, urban planning, marketing, telecommunication planning, disaster management, etc.

In the fully automated approach, the system itself detects the objects such as buildings, trees, roads, etc. in an image. In semi-automated approach the user manually selects the objects in an image. So, except the detection part remaining procedure for generating $3 \mathrm{D}$ city model in automated and semi-automated systems is the same. Hence, the modules developed in our approach can be used in developing automated $3 \mathrm{D}$ city model generation.

\section{A. Scope for further work}

- To have a real world scene like looking of 3D city model, Pattern Matching is to be done.

- Buildings are classified into two kinds. Convex shaped and Concave shaped structures. For Concave shaped buildings like ' $U$ ' shaped, 'V' shaped, etc., Semi- automated approach needs more number of interactions from user. So implementing Delaunay triangulation method may reduce interactions per building.

- Delaunay triangulation draws extra lines i.e. lines that do not belong to a building. We should remove those lines. Automatically removing them will be an efficient work.

- Coming to visualization we have developed grid values of buildings that are rectangular shaped only. Generating grid for all regular and irregular shapes (triangular, spherical, trapezium, etc.) looks very effective and therefore very sophisticated city models can be easily built from this 'vtk' grid file.

- 3D models of buildings are developed assuming same height for all buildings. If height data such as DEM files are available we can generate more effective and realistic 3D City Models.

\section{ACKNOWLEDGMENTS}

The present work is based on the M. Tech project work done by the first author at ANURAG (DRDO), Hyderabad. The authors are grateful to the Director, ANURAG and Mr. Dilip Kumar Dalei, Mr. Ravi kumar and Mr. B V H K Nanada, Scientists in ANURAG, DRDO for their permission to use available resources and their guidance throughout this work. The first author is thankful to Prof. L. Siva Reddy (Vice principal \& HOD, CSE Department) and to the Principal for providing all the facilities during his studies at CVR college of Engineering.

\section{REFERENCES}

[1] Y. Takase, N. Sho, A. Sone, K. Shimiya. Automatic Generation of 3D city Models and related Applications. International Archives of photogrammetry, remote sensing and Spatial Information Sciences, Vol. XXXIV-5/W10.

[2] Zuxun Zhang, Jun Wu, Yong Zhang, Yongjun Zhang, Jianquing Zhang. Multi-View 3D city model generation with Image sequences. International Archives of photogrammetry, remote sensing and Spatial Information Sciences, Vol. XXXIV-5/W12.

[3] "Study on making City Model with Image and Laser Range Data" (Paper Presented at the $22^{\text {nd }}$ Asian Conference on Remote Sensing, Singapore) Masafumi, Nakagawa.

[4] Yoshiaki, Kagawa, 2001. Automatic acquisition of 3D city data with air-borne TLS (Three Line Scanner) and Laser scanner.

[5] Brenner, C., Haala, N. and Fritsch, D, 2001. Towards fully automated $3 \mathrm{D}$ city model generation. In Automatic Extraction of Man-Made Objects from Aerial and Space Images III. 2001.

[6] Grun, A., Wang, X., 1998. CC Modeler: A Topology Generator for 3-D City Models. ISPRS Commission IV Symposium on "GIS - Between vision and application", Stuttgart/Germany, IAPRS. Vol. 32, Part 4, pp. 188-196.

[7] Construct 3D City Model by Multi-Sensor Data. Fei Deng, Zuxun Zhang, Jianqing Zhang. Remote Sensing School, Wuhan University, Wuhan, China-dengfwh@163.com

[8] Henricsson O., Bignone F., Willuhn W., Ade F., Kübler O., Baltsavias E., Mason S., Gruen A., 1996. Project AMOBE: Strategies, Current Status and Future Work. Paper presented at the 18. ISPRS Congress, 9. - 14. July, Vienna, Austria. In IAPRS, Vol. 31, Part B3, pp. 321 - 330.

[9] Maas, H.-G., 1999. Closed solutions for the determination of parametric building models from invariant moments of airborne laser scanner data. ISPRS Conference 'Automatic Extraction of GIS Objects from Digital Imagery, München/Germany. 